\title{
THE EFFECTS OF HYPNOSIS AND HYPNOTIC SUGGESTIONS ON THE MISMATCH NEGATIVITY IN HIGHLY HYPNOTIZABLE SUBJECTS
}

Hiltunen, Seppo

2019-04-03

Hiltunen , S , Virta , M , Kallio , S \& Paavilainen , P 2019 , ' THE EFFECTS OF HYPNOSIS AND HYPNOTIC SUGGESTIONS ON THE MISMATCH NEGATIVITY IN HIGHLY HYPNOTIZABLE SUBJECTS ' , International Journal of Clinical and Experimental Hypnosis , vol. 67 , no. 2 , pp. 192-216 . https://doi.org/10.1080/00207144.2019.1580966

http://hdl.handle.net/10138/313873

https://doi.org/10.1080/00207144.2019.1580966

acceptedVersion

Downloaded from Helda, University of Helsinki institutional repository.

This is an electronic reprint of the original article.

This reprint may differ from the original in pagination and typographic detail.

Please cite the original version. 


\section{The effects of hypnosis and hypnotic suggestions on the mismatch negativity (MMN) in highly hypnotizable subjects}

\section{Short title: \\ The effects of hypnosis and suggestions on MMN}

Seppo Hiltunen ${ }^{1,2 *}$, Maarit Virta ${ }^{2}$, Sakari Kallio $^{3,4} \&$ Petri Paavilainen $^{2,5}$

${ }^{1}$ Teaching and Learning Services, University services, University of Helsinki, Finland

${ }^{2}$ Department of Psychology and Logopedics, Faculty of Medicine, University of Helsinki, Finland

${ }^{3}$ Department of Cognitive Neuroscience and Philosophy, School of Bioscience, University of Skövde, Sweden

${ }^{4}$ Centre for Cognitive Neuroscience, University of Turku, Finland

${ }^{5}$ Cognitive Brain Research Unit, Department of Psychology and Logopedics, University of Helsinki, Finland

* Corresponding author

E-mail: seppo.hiltunen@helsinki.fi 


\section{Abstract}

The neural mechanisms associated with hypnosis were investigated in a group of nine highly hypnotizable subjects by measuring the mismatch negativity (MMN) component of the auditory event-related potential (ERP). ERPs were recorded using a passive oddball paradigm to sinusoidal standard and deviant tone stimuli of 500 and $520 \mathrm{~Hz}$, respectively, in four conditions: 1) pre-hypnosis, 2) neutral hypnosis, 3) hypnotic suggestion for altering the tone perception, and 4) post-hypnosis conditions. Earlier studies have indicated that hypnosis and hypnotic suggestions might have an effect on MMN, but the results of our study contradict these previous results: no statistically significant differences were found in the MMN amplitudes between the conditions.

keywords: hypnosis, suggestions, MMN, EEG 


\section{Introduction}

Hypnosis has been defined as "a state of consciousness involving focused attention and reduced peripheral awareness characterized by enhanced capacity for response to suggestion" (Elkins, Barabasz, Council, \& Spiegel, 2015). The experience of a hypnotic state is typically characterized by focused attention, an increase in absorption, lack of attention to extraneous stimuli, and a reduction in spontaneous thoughts (Oakley \& Halligan, 2009).

The brain mechanisms associated with hypnosis are still quite unclear, but several studies have reported activity changes in the prefrontal cortex and anterior cingulate cortex (Egner, Jamieson, \& Gruzelier, 2005; McGeown, Mazzoni, Venneri, \& Kirsch, 2009; Rainville, Hofbauer, Bushnell, Duncan, \& Price, 2002). Some studies have shown changes in the functional connectivity of the brain (Fingelkurts, Fingelkurts, Kallio, \& Revonsuo, 2007a; Hoeft et al., 2012; Jamieson \& Burgess, 2014), decreased activity in the default mode network (Deeley et al., 2012; Demertzi et al., 2011; McGeown et al., 2009) and increased activity in the prefrontal attentional system (Deeley et al., 2012) after hypnotic induction. The neuroimaging results of hypnosis studies have been summarized in the recent reviews by Landry, Lifshitz, and Raz (2017), De Benedittis (2015) and Vanhaudenhuyse, Laureys, and Faymonville (2014). Furthermore, Terhune, Cleeremans, Raz, and Lynn (2017) have provided a synthesis of the current knowledge regarding top-down regulation of human consciousness and perception in hypnosis. They concluded that the specific cognitive mechanisms and roles of different cortical and subcortical regions in the implementation of top-down control serving responsiveness to hypnotic suggestions remain poorly understood.

The most common method used in investigating the brain mechanisms of hypnosis is electroencephalography (EEG). EEG has an excellent temporal resolution, allowing changes in oscillatory brain activity to be monitored. Consequently, EEG rhythms in hypnosis and the waking state have been the focus of interest in several studies. The fractal dynamics of EEG rhythms have been found to be more random and less correlated in hypnosis upon auditory stimulation (Lee \& Koo, 2012). EEG band wave activity and global functional connectivity have also varied as a function of hypnotizability (Cardena, Jonsson, Terhune, \& MarcussonClavertz, 2013). Jensen, Adachi, and Hakimian (2015) have summarized the recent research results on the associations between hypnosis and brain oscillations. They have introduced a theta-gamma oscillation model of hypnosis, where hypnosis is most closely linked to power 
in the theta band $(4-8 \mathrm{~Hz})$ and hypnotic responding may be associated with changes in the timing of gamma oscillations. They also discuss the shortcomings of the EEG method, such as the spatial restrictions of the method and its limitations in capturing the highest gamma activity.

In addition to EEG oscillations, event-related potentials (ERPs) have been exploited in studies on the brain mechanisms of hypnosis. ERPs are tiny changes in brain activity that can be extracted from the EEG data by signal-averaging techniques. With millisecond resolution, the method enables researchers to monitor sensory, cognitive and motor processing in the brain. A widely-studied sensory ERP component is the mismatch negativity (MMN), which reflects the processing of changes in auditory stimulation (Näätänen, Paavilainen, Rinne, \& Alho, 2007). MMN is usually recorded in a so-called oddball-paradigm, where "deviant" stimuli (e.g., tones of a different pitch) are randomly presented among a train of frequent physically identical "standard" stimuli (on paradigms for MMN measurements, see e.g., Näätänen, Pakarinen, Rinne, and Takegata, 2004). MMN appears as a fronto-central, negative deflection in the deviant-stimulus ERP, usually peaking ca. 100-250 ms after the onset of the deviant stimulus (Näätänen et al., 2007). MMN is a relatively automatic and attentionindependent brain response to a stimulus change (Sussman, 2007). It is elicited even when the subject's attention is directed away from the auditory stimuli, for instance, they are watching a silent video during the auditory stimulation. In spite of the large degree of automaticity of the MMN response, top-down effects on the MMN have also been reported (for a review, see Sussman, 2007). For example, Sussman, Winkler, Huotilainen, Ritter and Näätänen (2002; see also Sussman, 2013) observed that when subjects were presented with identical tone patterns and instructed to assess them in two different ways, requiring either discrimination of pattern or pitch, the MMN to deviant stimuli was generated according to the task demands. MMN amplitude changes related to highly-focused attention (e.g., Woldorff, Hillyard, Gallen, Hampson \& Bloom, 1998) or even to concurrent motor actions (Tiainen, Tiippana, Paavilainen, Vainio, \& Vainio, 2017) have also been reported. Thus, although the generation of MMN is largely independent of attention, top-down effects can modulate the strength of the response.

Theoretical explanations of MMN have developed from the original sensory-memory trace interpretation (Näätänen, Gaillard, \& Mäntysalo, 1978) towards the regularity violation-based interpretation (Winkler, 2007). According to the latter account, the auditory system 
constantly creates representations of the regularities embedded in the auditory environment. Incoming stimuli are compared with temporally aligned predictions, and if the predictions are violated, the $\mathrm{MMN}$ is elicited, reflecting the updating of the regularity representations. It has also been proposed that the MMN signal is transmitted to the frontal lobes, which may in turn elicit an involuntary attention switch to the stimulus change (Escera, Yago, \& Alho, 2001; Näätänen et al., 2007). Hence, the MMN mechanism automatically alerts of potentially important changes in the unattended auditory environment and directs attentional resources for their further processing. Sources of MMN have been localized in the temporal lobe on the auditory cortices, with an additional generator in the frontal cortices (Alho, 1995). The former areas may be involved in the regularity extraction and change-detection processes, whereas the frontal areas are associated with the attention-switch mechanisms (Rinne, Alho, Ilmoniemi, Virtanen, \& Näätänen, 2000). The involuntary attention switches are manifested in the P3a component, often following the MMN, especially to salient deviants (see e.g., Alho, Winkler, Escera, Huotilainen, Virtanen, Jääskeläinen, Pekkonen \& Ilmoniemi, 1998; for a review, see Escera \& Corral, 2007).

MMN reflects such basic functions of the central auditory system that it is elicited even during REM sleep (Atienza, Cantero, \& Dominguez-Marin, 2002; Sculthorpe, Ouellet, \& Campbell, 2009) and in patients soon waking from coma (Daltrozzo, Wioland, Mutschler, \& Kotchoubey, 2007). However, MMN amplitude has been shown to decrease under sleepiness and drowsiness conditions (Paavilainen et al., 1987; Sallinen \& Lyytinen, 1997) or in the case of mental fatigue (Yang, Xiao, Liu, Wu, \& Miao, 2013). Consequently, the arousal level has an effect on MMN.

Terhune et al. (2017) describe hypnosis as a unique form of top-down regulation, where higher (e.g., frontal) brain areas exert an influence on processing at the lower levels. They review a multitude of studies where hypnosis has been successfully used, for instance, to produce auditory and visual hallucinations in highly hypnotizable individuals. Consequently, it would be interesting if such hypnotic top-down effects could also modulate the auditory information processing reflected by MMN. However, there are only a few studies on the effects of hypnosis on the MMN. In a study with a single highly hypnotizable subject, Kallio, Revonsuo, Lauerma, Hämäläinen, and Lang (1999) observed that the MMN amplitude to a pitch change was larger in neutral hypnosis than in the pre-hypnosis condition. In another single-subject study, the same subject had a diminished MMN amplitude to the pitch change 
when she was performing both visual and audiovisual hypnotic hallucinations (Kallio, Revonsuo, \& Lang, 2005). Jamieson, Dwivedi, and Gruzelier (2005) recorded MMNs in 11 low- and 12 high-hypnotizable subjects in three conditions: pre-hypnosis, neutral hypnosis and post-hypnosis. Frontal MMN amplitude increased during neutral hypnosis and then dropped in the post-hypnosis condition for both hypnotizability groups.

The recent study by Facco et al. (2014) was the first and thus far ostensibly the only published multiple-subject study investigating the possible effect of hypnotic suggestions on the MMN. Their suggestions were intended to alter their subjects' perception of the auditory stimuli and to create a kind of amusia (a condition in which the individual is unable to recognize melodies or rhythms). This was carried out by removing the subjects' ability to recognize deviant rhythms, that is, changes in sine tone durations of $50 \mathrm{~ms}$ for the standard and $100 \mathrm{~ms}$ for the deviant tones. The authors evaluated the effect of this "hypnotically induced amusia for rhythm" on the MMN in five highly and five non-highly hypnotizable subjects. MMN was recorded in the pre-hypnosis and the hypnotic suggestion conditions. MMN amplitude was significantly decreased during hypnotic amusia, but only in the highly hypnotizable subjects.

In the visual modality, some findings obtained with highly hypnotizable subjects also suggest that by using hypnotic suggestions, it is possible to influence preconscious or highly automatized information processing such as color perception (Kallio \& Koivisto, 2013; Koivisto, Kirjanen, Revonsuo, \& Kallio, 2013), but possibly only with some of the most hypnotizable individuals (Kallio, Koivisto, \& Kaakinen, 2017). Although the auditory and visual information processing systems are differently structured and located in the human brain, there may also be similarities in the effects of hypnotic suggestions between them.

The present study aims at providing further information on whether neutral hypnosis and hypnotic suggestions can have top-down effects on auditory processing mechanisms which are thought to be, at least to a large extent, outside of voluntary cognitive control. We studied whether hypnotic suggestions such as "all the sounds in the sequence sound exactly the same" could affect the MMN to the pitch deviants in the oddball sequence. We used highly hypnotizable persons as our subjects in order to maximize the possible effects of hypnotic suggestions on altering the auditory perception. We used pitch deviants, which are most commonly used in MMN studies and typically produce a strong MMN response (Kujala, Tervaniemi, \& Schroger, 2007). Pitch deviants were also recommended by Facco et al. 
(2014) for further studies. The pitches of the standard and deviant tones $(500$ and $520 \mathrm{~Hz}$, respectively) were chosen to be closer to each other than in the Kallio et al. (1999) study (500 $\mathrm{Hz}$ and $553 \mathrm{~Hz}$ ) for several reasons. If the pitch difference between the standard and deviant tones is large, the auditory N1 component to deviants is also enhanced: The repetitive standard stimulus drives the auditory-cortex neurons specific to their pitch in a partially refractory state whereas the deviants will stimulate a fresh, strongly-reacting population of neurons. The resulting N1 enhancement may summate over the MMN, making it difficult to separate these two components (Kujala et al., 2007). By using a rather small (but still clearly observable) pitch difference, we also tried to minimize the involuntary attention switching toward the deviants that might reduce the depth of hypnosis and impair the influence of the suggestions ("all tones sound similar"). In order to avoid excessive EEG alpha band activity (which is typical when relaxed and with eyes closed) contaminating the ERPs, our subjects watched a silent monotonous video.

Four test conditions were included: pre-hypnosis, neutral hypnosis, hypnotic suggestion and post-hypnosis. None of the previous hypnosis MMN studies had included all four conditions in the same experiment. Thus, this is the first study allowing a direct comparison of MMN, for instance, between neutral hypnosis and hypnotic suggestion conditions. On the basis of previous studies (Facco et al., 2014; Jamieson et al., 2005; Kallio et al., 2005; Kallio et al., 1999), we hypothesized that (Hypothesis 1) the MMN amplitudes will differ between the conditions. More specifically, we further hypothesized that the MMN amplitude is (2) increased in the neutral hypnosis condition compared with the pre-hypnosis condition, (3) diminished in the hypnotic suggestion condition compared with the pre-hypnosis and neutral hypnosis conditions, and (4) there will be no statistically significant MMN amplitude differences between the post-hypnosis and pre-hypnosis conditions. The possible findings may also have useful clinical implications, for example by increasing our understanding of the extent to which processes in the patient's brain can be influenced by hypnotherapy.

\section{Methods}

\section{Subjects}


The subjects were recruited through advertisements in the mailing lists of students of psychology and educational sciences at the University of Helsinki. The inclusion criteria for the hypnotizability measurements were as follows: (1) 18-45 years of age, (2) no diagnosis of psychosis or bipolar disorder, (3) no current severe depression, and (4) no neurological disorders, apart from migrane, were allowed. In total, 57 subjects reserved a place in the hypnotizability measurement group sessions. The potential subjects completed a questionnaire to ensure their suitability for the study and brought it with them to the hypnotizability measurement session. In the questionnaire, they provided detailed information about their education, work, health and medication. No individuals were excluded for not meeting the inclusion criteria. Nine persons did not show up for the group session and did not cancel their participation beforehand, so a total of 48 participated.

The hypnotizability of the 48 subjects was measured using the Finnish version (Kallio, 1996; Kallio \& Ihamuotila, 1999) of the Harvard Group Scale of Hypnotic Susceptibility, Form A (HGSHS:A) (Shor \& Orne, 1962). A subgroup of highly hypnotizable subjects (having HGSHS:A hypnotic susceptibility score of nine or more) was subsequently selected for the experiment with the ERP recordings. Total of nine highly hypnotizable subjects were identified, all of whom consented to participate in the ERP measurement.

The highly hypnotizable subjects ( 1 male, 8 females; mean age: 25.7 years, sd: 5.1 , range: 20-37 years; HGSHS:A score mean: 10.1, sd: 0.9, range: 9-11) were students with an average education history of about 16.1 years (sd: 2.8, range: $12.5-19$ years). One subject had previously been diagnosed with depression, but had subsequently recovered. One subject had an ADHD diagnosis, one had a diagnosis of migraine, and one reported having had migraine symptoms but without a diagnosis. One subject reported using psychotropic medication (Gabapentin $300 \mathrm{mg}$ ) for nerve pain at the time of the hypnotizability measurement, but had no further need for medication at the time of the ERP measurements. The time between the hypnotizability measurement session and the ERP measurement session varied between 56243 days (mean 139.2 days, sd: 61.2). The relatively long time lag between these two measurements should not pose a problem as the stability of the measured hypnotizability has been proven to last for a period of at least two decades (Piccione, Hilgard, \& Zimbardo, 1989).

The study was approved by the University of Helsinki Ethical Review Board in the Humanities and Social and Behavioural Sciences and performed in accordance with the 
ethical standards of the Declaration of Helsinki. All subjects gave their written informed consent prior to participating in the study.

\section{Stimuli}

The ERPs were measured in an oddball paradigm using pure sinusoidal tones (duration 100 ms with linear $10-\mathrm{ms}$ rise and fall times). Standard tones $(500 \mathrm{~Hz} ; \mathrm{p}=.82)$ and deviant tones $(520 \mathrm{~Hz}, \mathrm{p}=.18)$ were presented in a random order in blocks of 737 stimuli with a $400-\mathrm{ms}$ interstimulus interval (ISI) so that there were 2-7 standard tones between each deviant tone. 10 standards were presented at the beginning of each stimulus block. One block per condition was presented, each block lasting 6 min 8 seconds. The stimuli were presented from two loudspeakers (OWI Inc., CA), positioned on the left and right side of the subject about 100$110 \mathrm{~cm}$ from the subject with an opening angle about $160-170^{\circ}$. The intensity of the stimuli was about $56 \mathrm{~dB}$ SPL at the subject's ear level. Intensity was intended to be low enough not to disturb the subject's hypnotic state, but high enough be able to produce MMN.

\section{Procedure}

During the four experimental conditions, the subject was seated in a comfortable reclining chair in an electrically and acoustically shielded room and instructed to watch a silent nature video of a calmly flowing narrow forest river. The video was commercially sold for relaxation purposes (OutpostFX AB, www.outpostfx.com). A video that included only the monotonic movement of water was chosen in order to prevent the subject from becoming too alert and awakening from hypnosis during the experiment. The video was shown on an 18-inch display, located in front of the subject at a distance of about 140-150 cm from the subject's eyes. The experimenter (MV, who administered hypnosis in all of the experiments) sat to the right of the subject, so that she was able to see the subject's face sideways in order to observe when he/she closed/opened his/her eyes, for example, and to be able to modify suggestions and instructions accordingly.

Before the experiment got underway, the subject was told that his/her task in all of the experimental conditions was just to relax and watch the video, without paying any attention to 
the tones, and to avoid excessive blinking during the measurement session, if possible. The subject was not informed about the specific aim of the study and the expected effects of hypnosis.

The experimentee was then subjected to the four different experimental conditions. At the beginning and in the end of each condition, the experimenter asked the subject to provide a subjective evaluation of the experienced depth of hypnosis on a scale of 0 to $10(0=$ normal wake state, no hypnosis at all; $10=$ maximum possible depth of hypnosis experienced). During each condition, one block of the auditory stimuli described above was presented to the subject. The experimental conditions were presented in the following order:

1) Pre-hypnosis condition (henceforth PrH): The subject was just instructed to watch the video.

2) Neutral hypnosis condition (HY): Before presenting the auditory stimuli, a hypnotic induction was carried out in a structured way, while allowing for some personal modification (e.g., the time of closing the eyes). The induction consisted of eye fixation, closing eyes, relaxation and deepening of hypnosis by counting, and took around eight minutes to carry out. The subject was subsequently asked to open their eyes and start watching the video. The auditory stimulus block was the delivered. Once during the auditory stimulation $(\sim 3.5 \mathrm{~min}$ from the beginning), the experimenter gave a few more suggestions for intensifying the depth of hypnosis: You are staying in deep hypnosis and you may even go into deeper hypnosis...deeper hypnosis. When the stimulus block ended, the experimenter asked the subject to close their eyes.

3) Hypnotic suggestion condition (SU): The experimenter first made the following suggestions (translated from Finnish) to the subject who was still in hypnosis after the HY condition. The suggestions were intended to alter the subject's perception of the tone stimuli:

While watching the video, you may have heard beeping sounds in the background, although you did not need to pay attention to them at all. There is no need to pay attention to them in the next condition either. When the condition begins the next time, all the beeping sounds in the environment sound similar. They are just in the background as if they are meaningless and muffled... all the beeping sounds sound exactly the same in pitch...without meaning, soft in the background, with a similar pitch,... beeping sounds are in the background softly, and meaninglessly, all the beeping sounds have a similar pitch... When I ask you to open your eyes, you can do so easily, your hypnotic trance is not in any way disturbed and you will stay 
in deep hypnosis. You can easily focus on watching the video, you can give your full attention to the video.

The subject was then asked to open their eyes and watch the video. Again an auditory stimulus block was delivered. Once during the stimulus presentation $(\sim 3.5 \mathrm{~min}$ from the beginning), the experimenter made a few more suggestions for intensifying the altered tone perception and the depth of hypnosis:

You remain in deep hypnosis and you may even go into deeper hypnosis. Beeping sounds are playing in the background softly, and meaninglessly...all the beeping sounds are the same and have a similar pitch. You can easily keep your eyes open, and your hypnotic trance is not disturbed by it. You may even go into deeper hypnosis and you can still give your full attention to the video.

When the stimulus block ended, the experimenter asked the subject to close their eyes and a hypnotic reversal procedure was administered. This included counting backwards from 10 to 1 with suggestions about waking and returning to a normal waking state, with special emphasis on the normalization of the tone perception. During the procedure, the subject opened their eyes.

4) Post-hypnosis condition (PoH): The subject was instructed to watch the video and the last auditory stimulus block was delivered.

After the final condition, the experimenter interviewed the subject about their experiences during the experiment. The experimental procedure (four conditions, including induction, suggestions, termination and so forth) lasted about 45 minutes. The whole experiment, including the preparations for ERP recordings (fitting the cap and electrodes) and cleanup (removing the cap/electrodes, hair washing) lasted from one hour 45 minutes to two hours.

\section{EEG recording and ERP averaging}

The EEG was recorded with a Biosemi measurement system (www.biosemi.com, 0-102,4 Hz bandpass, $512 \mathrm{~Hz}$ sampling rate) with a 64-channel cap from the same manufacturer. In addition, separate electrodes were attached to the left and right mastoids and to the tip of the nose. Eye movements were monitored with electrodes on the right and left canthi, and below the left eye. During the EEG recording, the nose served as the reference electrode. The 
grounding electrode (CMS) was attached to the back of the head (according to Biosemi standard layout). ERPs to the standard and deviant tones were averaged with MATLAB R2016 using an EEGLAB toolbox (www.sccn.ucsd.edu/eeglab). The EEG was filtered with a 0.5-30 Hz bandpass, re-referenced to the average of the right and left mastoid electrodes (to provide maximum-amplitude MMNs) and cut to epochs starting $100 \mathrm{~ms}$ before the onset of the tone and ending $500 \mathrm{~ms}$ after the onset. Epochs containing voltage changes (e.g., artefacts related to eye movement or muscle tension) that were too large (over $\pm 100 \mu \mathrm{V})$ were rejected. The remaining epochs were averaged to obtain the ERPs to standard and deviant tones, separately in each experimental condition. The 100-ms pre-stimulus period served as the baseline for ERP amplitude measurements. The difference waveforms were calculated by subtracting the standard-tone ERPs from those to the deviant tones.

The MMNs were measured by calculating the mean amplitudes from the standard and deviant-stimulus ERPs at Fz, F3 and F4 electrodes (where the MMN was largest) during 150-250 ms. This latency window was visually selected from grand-average difference waves. One subject exhibited a lot of eye blinks during the EEG recordings and in order to obtain reliable ERPs, Independent Component Analysis (ICA) for eye movement artifact correction was used. For this subject, we visually identified one to two well-characterized ICA components for eye blinks and lateral eye movements. We used visual inspection of component scalp maps, power spectrum, and raw activity to select and reject these artefactual ICA components. For another subject, an electrode with a bad contact had to be interpolated from the other surrounding electrodes using the EEGLAB toolbox.

\section{Statistical analysis}

The MMN mean amplitude (the dependent variable) was analyzed with a $2 \times 3 \times 4$ repeated measures analysis of variance, the factors being stimulus type (standard/deviant), electrode (F3, Fz, F4) and condition (PrH, HY, SU, PoH). F3 and F4 were included in the analysis in order to reveal possible hemisphere differences in the MMN amplitudes. The subjective hypnosis depth values were analyzed with repeated measures analysis of variance with the post hoc tests including Bonferroni corrections. The Greenhouse-Geisser correction was applied to all of the degrees of freedom of the F-tests. The significance level was set at $\mathrm{p}<$ .05 . 


\section{Results}

Figure 1 presents the ERPs at Fz to standard and deviant stimuli in the four experimental conditions (PrH, HY, SU, PoH). The ERPs to deviants were negatively displaced relative to those to standards in all conditions, starting at about $100 \mathrm{~ms}$. This negative displacement is the MMN.

(Figure 1 about here)

Figure 2 shows the deviant minus standard difference waves, enabling the comparison of MMN amplitudes and latencies between the conditions. The MMN peaked at approximately $200 \mathrm{~ms}$ and its onset and offset latencies were rather similar between the conditions. The MMN peak amplitude was largest in the $\mathrm{PrH}$ and lowest in the $\mathrm{PoH}$ condition. As a trend, the MMN seemed to decrease in successive conditions, although the amplitudes in SU and HY conditions were rather similar. No clear P3a component, following the MMN, was observed in any condition.

(Figure 2 about here)

As the MMN peaked in all conditions at around $200 \mathrm{~ms}$, the MMNs were measured for the statistical analyses from the difference waves as their mean amplitudes during 150-250 ms. The mean amplitudes at $\mathrm{Fz}$ and their standard deviations were as follows: $\mathrm{PrH}:-3.1 \mu \mathrm{V}(1.3)$, HY: $-2.1 \mu \mathrm{V}(0.7), \mathrm{SU}:-2.3 \mu \mathrm{V}(1.9)$ and $\mathrm{PoH}:-1.4 \mu \mathrm{V}$ (1.5). The $2 \times 3 \times 4$ repeated-measures ANOVA showed that the main effect of stimulus type (standard/deviant) was statistically significant $\left[F(1,8)=59.19, p<0.001\right.$, partial eta squared $\left.\left(\eta p^{2}\right)=0.88\right]$, indicating that a statistically significant MMN was obtained (i.e., the standard and deviant wave amplitudes differed from each other during the MMN measurement window). However, the main effects of the condition $\left[F(2,16)=1.41, p>0.05, \eta p^{2}=0.15\right]$ or that of the electrode $[F(1,10)=$ $\left.1.59, p>0.05, \eta p^{2}=0.17\right]$ were not statistically significant. Most importantly, the stimulus type $\mathrm{x}$ condition interaction was not significant $\left[F(2,14)=2.97, p>0.05, \eta p^{2}=0.27\right]$. Thus, no statistical evidence for MMN amplitude differences between the conditions was found.

The experimenter asked the subjects for a subjective evaluation of the experienced depth of hypnosis during the experimental conditions. To obtain the depth values for the each condition, the average of the values reported at the beginning and at the end of each condition was calculated for each subject. The mean subjective hypnosis depth values and their 
standard deviations in the four experimental conditions are shown in Table 1. The repeatedmeasures ANOVA showed that subjective hypnosis depth values differed between the conditions $\left[F(2,12)=60.00, p<0.001\right.$, partial eta squared $\left.\left(\eta p^{2}\right)=0.88\right]$. The results of the post hoc tests between the conditions are also shown in Table 1.

(Table 1 about here)

After the experiment, the experimenter interviewed the subjects about their experiences during the conditions. Subjects' comments on experiencing sounds were rather diverse: Three subjects reported some changes in the perception of the sounds, noting, for example, that the sounds were more softer in the background (two subjects), or that the sounds disappeared (one subject). Three subjects reported having heard differences between the sounds, two reported that they had noticed no differences and one was not able to describe anything related to the sounds since they had paid no attention to them. In general, it was difficult for the subjects to recall or to connect their auditory experiences to the specific conditions.

Figure 3 shows the grand-average ERPs across those subjects $(\mathrm{N}=6)$, who had reported in the HGSHS:A hypnotizability group measurement that the "fly" suggestion (which can be classified as a "hallucinating suggestion") had come true. Our aim was to find out whether the hypnotic suggestions for altering perception of the sounds in the experiment might have had a stronger influence on the MMN amplitude in this subgroup, as they were evidently the most prone to auditory hallucinations. On visual inspection, the MMN peak amplitude indeed seems to be lowest in the SU condition but the differences compared to $\mathrm{HY}$ and $\mathrm{PoH}$ are marginal. The mean MMN amplitudes at Fz during 150-250 ms were as follows: PrH: -3.2 $\mu \mathrm{V}$ (1.5), HY: $-2.2 \mu \mathrm{V}(0.8), \mathrm{SU}:-2.0 \mu \mathrm{V}$ (2.0) and $\mathrm{PoH}:-1.9 \mu \mathrm{V}$ (1.5). The $2 \times 3 \times 4$ repeatedmeasures ANOVA showed that the main effect of stimulus type (standard/deviant) was again statistically significant $\left[F(1,5)=37.27, p<0.01, \eta p^{2}=.88\right]$, indicating that an MMN was obtained. However, the stimulus type $\mathrm{x}$ condition interaction was not significant $[F(2,9)=$ $1.47, p>0.05, \eta p^{2}=0.23$ ] even in this subgroup (however, one must take into account that due to the the small $\mathrm{N}$, a large effect is needed to reach statistical significance).

(Figure 3 about here)

\section{Discussion}


The main purpose of the present study was to investigate whether neutral hypnosis and hypnotic suggestions could have an influence on MMN in highly hypnotizable subjects. Our experimental setup was found to produce clear MMN responses. However, no statistically significant differences in the MMN amplitudes between the four conditions were found. Thus, we could not verify our Hypothesis 1 ("the MMN amplitudes will differ between the conditions") and, consequently, our next two hypotheses were not supported by the evidence either ("MMN amplitude is increased in the neutral hypnosis condition compared with the pre-hypnosis condition"; "MMN amplitude is diminished in the hypnotic suggestion condition compared with the pre-hypnosis or neutral hypnosis condition"). Only our fourth hypothesis ("there are no significant MMN amplitude differences between the post-hypnosis and pre-hypnosis conditions") was confirmed (although there was a visually observed trend that the MMN amplitude diminished in successive conditions).

We found no statistically significant influence of neutral hypnosis on the MMN amplitude. Our result differs from those of two earlier studies, where the MMN amplitude was found to increase during the neutral hypnosis compared to the pre-hypnosis condition (Jamieson et al., 2005; Kallio et al., 1999). Jamieson et al. (2005) had 11 low and 12 highly hypnotizable subjects in their ERP study. They found a slight increase in MMN amplitude in the neutral hypnosis condition, even in low hypnotizable subjects. This result was the opposite of that obtained in the present study, where, if anything, the MMN amplitude was smaller in the neutral hypnosis condition than in the pre-hypnosis condition.

Kallio et al. (1999) was a single-subject study but their stimulus parameters were rather similar to those in the present study (i.e., oddball paradigm, pitch deviant). However, we used a smaller pitch difference between the standard and the deviant to eliminate possible N1 contamination (see Kujala et al., 2007). Another difference was that Kallio et al. (1999) used a post-hypnotic (one word) command to induce hypnosis which may better maintain a higher alertness level in the subject. Their result was similar to that of Jamieson et al. (2005) multiple-subjects study: MMN amplitude increased in the neutral hypnosis condition. Interestingly, when referring on their unpublished data, Kallio et al. (2005) noted that only two of their 12 highly hypnotizable subjects had change in MMN amplitude in the neutral hypnosis condition compared to the pre-hypnosis condition.

We found no statistically significant influence of suggestions of altered perception on the MMN amplitude, contrary to Facco et al. (2014), who reported significantly diminished 
MMN amplitudes in highly hypnotizable subjects as a result of "amusia for rhythm". This amusia was induced in hypnosis by suggestions that the subjects "become completely deaf to tone duration". Their sounds were at the same pitch, but the deviants were longer in duration (100 ms) than the standard (50 ms).

The reasons for the discrepancy between Facco et al.'s (2014) results and ours are not clear. Our results were also obtained with highly hypnotizable subjects. The Facco et al.'s (2014) $\mathrm{HH}$ group may have mainly consisted of subjects who were quite experienced with hypnosis since the authors used a post-hypnotic command to induce hypnosis and hypnotic amusia, as did Kallio et al. (1999) with their hypnosis virtuoso. Mean of the subjectively evaluated hypnosis depth in the Facco et al.'s (2014) HH group was 8.2, and that of the present study subjects 5.8, which may indicate that their subjects were in deeper hypnosis than the subjects in the present study. However, there is no reliable objective method for evaluating depth of hypnosis and the subjective scale is highly prone to variation depending on how it is understood and used (for details see e.g., Radtke \& Spanos, 1981, 1982). Moreover, contextual variations may appear, for instance, depending on whether the subjects's eyes are open or closed during hypnosis.

In the visual inspection, the MMN peak amplitudes in the HY and SU conditions (see Figure 2) were very similar. Thus, there seems to be no extra effect related to the altered-perception suggestions between those two conditions. However, in the subgroup of six subjects who were capable of experiencing hypnotically induced hallucinations, the MMN amplitude was lowest in the SU condition (see Figure 3), although no statistically significant differences between the MMN amplitudes were found (possibly due to the small sample size). Tentatively, this result suggests that in future MMN studies, one should only use subjects who are capable of experiencing hypnotically induced hallucinations.

There may also be certain interpretational difficulties in Facco et al.'s study. For example, they reported that no similar MMN amplitude reduction was observed in the group of low hypnotizable subjects (LH group; $\mathrm{N}=5$ ) as was found in the $\mathrm{HH}$ group. However, if one inspects their Figure 2 carefully, it seems that although the MMN peak amplitudes for the LH group (around $180 \mathrm{~ms}$ ) do indeed seem to be similar in the pre-hypnosis and the hypnotic suggestion conditions, there is actually a clear subsequent difference between the conditions during 230-280 ms. The size and scalp distribution of this effect are very similar to the MMN amplitude reduction effect reported for the $\mathrm{HH}$ group at the earlier latency. If this is a real 
MMN effect, just occurring for some reason at a later latency in the LH group than in the $\mathrm{HH}$ group, it may indicate that the mere suggestions were sufficient to produce the MMN amplitude reduction effect, and no hypnosis was needed: Their LH group was obviously in a less profound hypnotic state (hypnosis depth: 3.1) during the hypnotic suggestion condition. However, it is also possible that the late effect in the LH group data does not reflect MMN but rather some other, later ERP component elicited by the deviant stimuli. For example, it might be the so-called $\mathrm{N} 2 \mathrm{~b}$ component which is related to more conscious deviance processing than the MMN (Folstein \& Van Petten, 2008) and is usually elicited only when the stimuli are attended to. Unfortunately, Facco et al. (2014) did not report how their subjects were instructed to listen to the sounds or what they were doing during the conditions (e.g. whether their attention was directed to the sounds or elsewhere). Hence, it is difficult to estimate this possibility.

Another factor potentially complicating the interpretation of Facco et al.'s (2014) results was that they used duration deviants and their deviant stimuli were longer in duration than the standard stimuli. Since longer stimuli usually produce stronger exogenous ERP components as well (e.g. N1; Kujala et al., 2007), it is possible that in their deviant-stimulus ERPs the effects of true MMN and the overlapping N1 were somewhat confounded (this problem could have been avoided by using deviants that are shorter than standard). However, even if the MMN they recorded had not been totally "pure", it cannot explain away the suggestion effects they observed.

In addition to Facco et al. (2014) study, other data suggest the possible influence of hypnotic suggestions on the MMN. For example, Kallio et al. (2005) described an MMN experiment with a high hypnotizable subject engaged in a visual and an audio-visual hallucination task simultaneously as the subject was presented with sounds. They found that the MMN was diminished during hallucination when compared to the pre-hypnosis condition (which, however, was recorded on a different day, making the comparison somewhat unreliable).

We could speculate as to why no statistically significant MMN differences were found between the different conditions of the present study. First, MMN might, after all, reflect such low-level, "hard-wired" and preattentive brain mechanisms that it cannot be influenced by top-down hypnotic suggestions. Interestingly, the results of the recent meta-analysis by Landry et al. (2017) do not support the frontal top-down theories of hypnosis either. More research is definitely needed to resolve when, where and how hypnosis may affect the 
"bottom-up" or lower-level type of functionality in the human brain (including the executive, salience and default networks; see Landry et al., 2017).

Second, if all of our subjects were not immersed in deep enough (or maximum) hypnosis during our hypnosis conditions, it may have influenced the results. There may be quite a lot of inter-individual variation in the effects of hypnosis, probably related to differences in hypnotizability. Our subjects were "naïve", in the sense of not having much previous experience of hypnosis, whereas Kallio et al. (1999) used a very experienced "hypnosis virtuoso" and Facco et al.'s (2014) subjects were experienced in hypnosis. Our subjects' 0-10 evaluations concerning the depth of their hypnosis were, on average, 5.8 (HY) and 5.7 (SU), so there is still some room for deepening the hypnosis. We used a "standardized" induction procedure for all subjects, and there was no extra individual deepening of induction in addition to the normal 1 to 10 counting. In order to maintain the subjects in deep hypnosis, we nonetheless added extra suggestions in the middle of the hypnosis conditions (HY and $\mathrm{SU})$. The subjective hypnosis depth evaluations showed a significant statistical difference between the non-hypnosis (PrH and $\mathrm{PoH}$ ) and hypnosis (HY and $\mathrm{SU}$ ) conditions, and they did not differ between the PrH and the PoH or between the HY and the SU conditions. Some (4/9) of our subjects reported that opening their eyes influenced their hypnosis, while about half of the subjects said that their hypnosis had been deeper in the group session where their hypnotizability was measured than during the actual experiment. However, the aforementioned problems in the subjective evaluations concerning the depth of hypnosis must be kept in mind when interpreting these reports.

Third, despite the depth of the hypnosis in general, the hypnotic suggestions for altering the perception of the sounds may not have altered the phenomenological experience for most of the subjects. This was difficult to evaluate after the experiment, since the subjects were explicitly instructed at the outset that there was no need to pay any attention to the sounds. What is more, due to the nature of hypnosis, the exact recalling of experiences afterwards is typically difficult, especially with regard to the point in time during the experiment - for example in which condition - the specific details occurred. By asking the subjects about their perceptions of the sounds during the experiment, their attention would have inevitably been directed to the sounds, a phenomenon that we wanted to avoid in order to record as pure an MMN as possible. The expectations of the subjects toward hypnosis and hypnotic suggestions may also have an influence on how suggestions are realized: some may expect 
the suggestions to be realized automatically, while others may give their imagination free rein in order to realize the suggestions.

Forth, we could also speculate whether the two subjects with an ADHD or a migraine diagnosis might have skewed our results. A meta-analysis of MMN studies with children having ADHD reported reduced MMN amplitudes compared to healthy controls (Cheng, Chan, Hsieh, \& Chen, 2016). Our subject with an ADHD diagnosis had MMN amplitude values in the PrH condition that were very close to the group average. Omitting this subject from the statistical analyses did not alter the results. Migraine has not been shown to affect the MMN (Morlet, Demarquay, Brudon, Fischer, \& Caclin, 2014). Another study has reported a slight increase in the latency of MMN (de Tommaso et al., 2004). We, however, used a wide (150-250 ms) window for calculating the MMN mean amplitudes, so possible slight latency changes are unlikely to have affected the results. It should also be noted that these two subjects were not included in the aforementioned results of the special group of subjects $(\mathrm{N}=6)$ who had experienced the "fly suggestion" in the hypnotizability measurements.

Fifth, the finding that our results contradict previous studies could also be related to the other differences in the experimental setup, stimuli and conditions. In particular, for reasons presented in the Introduction, we wanted to use a relatively small pitch difference between the standard and deviant stimuli. However, we deem it unlikely that it could have contributed to the absence of MMN differences between the conditions. The pitch difference used was still easily distinguishable and it produced clear and robust MMN responses (for MMNs to small pitch differences, see e.g., Sams, Paavilainen, Alho and Näätänen, 1985). On the other hand, the absence of the P3a component (see Escera \& Corral, 2007) in the present data indicates that we succeeded in our aim to select a pitch difference small enough to prevent extensive involuntary attention switching to deviant stimuli.

The decreasing trend in the MMN amplitudes in successive conditions (if reflecting a true effect) may be caused by the subjects getting tired, and their alertness level decreasing during the rather monotonous video-viewing task. The lowest MMN amplitudes were observed in the last $(\mathrm{PoH})$ condition. The MMN is known to be sensitive to variation in arousal level and fatigue (Paavilainen et al., 1987; Sallinen \& Lyytinen, 1997; Yang et al., 2013). Furthermore, the "standard" induction procedure may increase the risk of decreasing the alertness level, compared to an induction administered by a post-hypnotic command. Induction by a post- 
hypnotic command, however, was not a reasonable option in our case since we used subjects with no experience of hypnosis.

Another factor that may have contributed to the progressively decreasing MMN amplitudes might have been increased mind wandering. Our video-viewing task was quite dull: just a calmly flowing river, without any other actions to pay attention to. Using a video task with a low cognitive load might be more likely to ensure that the subjects remain under deep hypnosis. On the other hand, this kind of task may have increased the risk of mind wandering, especially in the final $(\mathrm{PoH})$ condition. On the basis of the definition of hypnosis (Elkins et al., 2015), subjects under hypnosis are in a state of focused attention and reduced spontaneous thoughts (i.e. in a reduced mind wandering state). In a previous study, it was found that MMN amplitude decreases during mind wandering (Braboszcz \& Delorme, 2011). As far as we know, there are no MMN studies concerning mind-wandering during hypnosis, but we can speculate that MMN could have been diminished in both of our hypnosis conditions (HY and SU) because of reduced alertness, but at the same time increased due to reduced mind wandering compared to the waking state. Suggestions in themselves (even if they are not directly related to altering the perception of tones) may also add to the cognitive/attentive load, which may affect MMN by diminishing it, as was possibly the case in Kallio et al.'s (2005) study on hallucinating.

As for the strengths of our study, we designed it to overcome certain shortcomings found in previous studies. As far as we can gather, this was the first MMN hypnosis study combining all four test conditions (pre-hypnosis, neutral hypnosis, hypnotic suggestion, and posthypnosis conditions) in the same study. We had a reasonably large sample of highly hypnotizable individuals as our subjects. Our experimental setup and stimulus parameters were designed in such a way that any possible contamination by other ERP components (e.g. $\mathrm{N} 1, \mathrm{~N} 2 \mathrm{~b}$ ) or resting state alpha band waves were minimized. Furthermore, the standard analysis procedures used in basic MMN studies were applied.

Our study naturally has its own limitations that should be considered when interpreting the results. We conducted our experiment only with a group of highly hypnotizable subjects, as we expected to obtain the clearest results with them (as previously found by Facco et al. (2014). In a retrospective evaluation, the lack of a control group of low-hypnotizable subjects in the present study was not such a serious shortcoming after all: as no statistically significant MMN effects were obtained with the highly hypnotizable subjects, it seems unlikely that such 
effects could have been found with low-hypnotizable subjects either. In our study, hypnotizability was measured once by using HGSHS:A. An individual assessment in addition to a group variant would have given a better estimate of the subjects' hypnotizability, and especially about their hallucinating ability. We decided to use HGSHS:A as it is commonly used and provides a good compromise when one has to screen a large number of subjects to find highly hypnotizable subjects for the study. In the future, there may also be a need to measure the hypnotizability of the subjects with open eyes in the standardized way.

One methodological compromise that inevitably had to be made in the present study was the lack of counterbalancing between different conditions. It is known that a person may not be in a similar alert state of consciousness after hypnosis as they were before (Fingelkurts, Fingelkurts, Kallio, \& Revonsuo, 2007b; Williams \& Gruzelier, 2001). This was also shown in the results of the single-subject study by Kallio et al. (1999), having counterbalancing between the baseline (pre-hypnosis) and neutral hypnosis conditions. Thus, if the experimental conditions are always presented in the same order, carry-over effects may occur, possibly affecting the results. In the present data, there was a trend towards the lowest MMN amplitudes being in the PoH condition. This may be due to a small carry-over effect after hypnosis (including a eight-minutes induction) or to a progressively decreasing alertness level, as discussed earlier. We could not counterbalance the different conditions as in a within-subject design with a restricted number of highly hypnotizable subjects available, it could not have been done in a satisfactory way. Our primary goal was to find out whether there is any indication of the effect of hypnosis and hypnotic suggestions on the MMN. In future studies, the possible effects of hypnosis and suggestion, alertness level and mind wandering should be better controlled, for instance by adding a control group of subjects that execute all four conditions in the waking state, namely without hypnosis and suggestions.

In conclusion, no statistically significant evidence for the effect of neutral hypnosis and hypnotic suggestion on the MMN amplitude in highly hypnotizable subjects was obtained. Since other studies have reported the effects of hypnosis and hypnotic suggestions on MMN, further carefully designed MMN studies, especially with highly hypnotizable subjects are required to resolve this issue.

\section{Acknowledgements}


SH received a grant from the Society of Scientific Hypnosis in Finland. The grant was used for the preliminary design and the first piloting phase of this study in 2014 .

We would like to thank Tommi Makkonen for his help in coding the experimental setup and analyzing the data.

\section{References}

Alho, K. (1995). Cerebral generators of mismatch Negativity (MMN) and its magnetic counterpart (MMNm) elicited by sound changes. Ear and Hearing, 16(1), 38-51. doi:Doi 10.1097/00003446-199502000-00004

Alho, K., Winkler, I., Escera, C., Huotilainen, M., Virtanen, J., Jaaskelainen, I. P., . . Il Imoniemi, R. J. (1998). Processing of novel sounds and frequency changes in the human auditory cortex: magnetoencephalographic recordings. Psychophysiology, 35(2), 211-224.

Atienza, M., Cantero, J. L., \& Dominguez-Marin, E. (2002). Mismatch negativity (MMN): an objective measure of sensory memory and long-lasting memories during sleep. International Journal of Psychophysiology, 46(3), 215-225.

Braboszcz, C., \& Delorme, A. (2011). Lost in thoughts: neural markers of low alertness during mind wandering. Neuroimage, 54(4), 3040-3047. doi:10.1016/j.neuroimage.2010.10.008

Cardena, E., Jonsson, P., Terhune, D. B., \& Marcusson-Clavertz, D. (2013). The neurophenomenology of neutral hypnosis. Cortex, 49(2), 375-385. doi:10.1016/j.cortex.2012.04.001

Cheng, C. H., Chan, P. S., Hsieh, Y. W., \& Chen, K. F. (2016). A meta-analysis of mismatch negativity in children with attention deficit-hyperactivity disorders. Neuroscience Letters, 612, 132-137. doi:10.1016/j.neulet.2015.11.033

Daltrozzo, J., Wioland, N., Mutschler, V., \& Kotchoubey, B. (2007). Predicting coma and other low responsive patients outcome using event-related brain potentials: a meta-analysis. Clinical Neurophysiology, 118(3), 606-614. doi:10.1016/j.clinph.2006.11.019

De Benedittis, G. (2015). Neural mechanisms of hypnosis and meditation. Journal of PhysiologyParis, 109(4-6), 152-164. doi:10.1016/j.jphysparis.2015.11.001

de Tommaso, M., Guido, M., Libro, G., Losito, L., Difruscolo, O., Sardaro, M., \& Puca, F. M. (2004). Interictal lack of habituation of mismatch negativity in migraine. Cephalalgia, 24(8), 663-668. doi:10.1111/j.1468-2982.2004.00731.x

Deeley, Q., Oakley, D. A., Toone, B., Giampietro, V., Brammer, M. J., Williams, S. C., \& Halligan, P. W. (2012). Modulating the default mode network using hypnosis. International Journal of Clinical and Experimental Hypnosis, 60(2), 206-228. doi:10.1080/00207144.2012.648070

Demertzi, A., Soddu, A., Faymonville, M. E., Bahri, M. A., Gosseries, O., Vanhaudenhuyse, A., . . . Laureys, S. (2011). Hypnotic modulation of resting state fMRI default mode and extrinsic network connectivity. Prog Brain Res, 193, 309-322. doi:10.1016/B978-0-444-53839$0.00020-\mathrm{X}$

Egner, T., Jamieson, G., \& Gruzelier, J. (2005). Hypnosis decouples cognitive control from conflict monitoring processes of the frontal lobe. Neuroimage, 27(4), 969-978.

Elkins, G. R., Barabasz, A. F., Council, J. R., \& Spiegel, D. (2015). Advancing research and practice: the revised APA Division 30 definition of hypnosis. International Journal of Clinical and Experimental Hypnosis, 63(1), 1-9. doi:10.1080/00207144.2014.961870 
Escera, C., \& Corral, M. J. (2007). Role of mismatch negativity and novelty-P3 in involuntary auditory attention. Journal of Psychophysiology, 21(3-4), 251-264. doi:10.1027/0269-8803.21.34.251

Escera, C., Yago, E., \& Alho, K. (2001). Electrical responses reveal the temporal dynamics of brain events during involuntary attention switching. European Journal of Neuroscience, 14(5), 877883.

Facco, E., Ermani, M., Rampazzo, P., Tikhonoff, V., Saladini, M., Zanette, G., .. S Spiegel, D. (2014). Top-down regulation of left temporal cortex by hypnotic amusia for rhythm: a pilot study on mismatch negativity. International Journal of Clinical and Experimental Hypnosis, 62(2), 129144. doi:10.1080/00207144.2014.869124

Fingelkurts, Fingelkurts, Kallio, \& Revonsuo. (2007a). Cortex functional connectivity as a neurophysiological correlate of hypnosis: an EEG case study. Neuropsychologia, 45(7), 14521462. doi:10.1016/j.neuropsychologia.2006.11.018

Fingelkurts, Fingelkurts, Kallio, \& Revonsuo. (2007b). Hypnosis induces a changed composition of brain oscillations in EEG: A case study. Contemporary Hypnosis, 24(1), 3-18. doi:DOI: 10.1002/ch.327

Folstein, J. R., \& Van Petten, C. (2008). Influence of cognitive control and mismatch on the N2 component of the ERP: a review. Psychophysiology, 45(1), 152-170. doi:10.1111/j.14698986.2007.00602.x

Hoeft, F., Gabrieli, J. D., Whitfield-Gabrieli, S., Haas, B. W., Bammer, R., Menon, V., \& Spiegel, D. (2012). Functional brain basis of hypnotizability. Arch Gen Psychiatry, 69(10), 1064-1072. doi:10.1001/archgenpsychiatry.2011.2190

Jamieson, G. A., \& Burgess, A. P. (2014). Hypnotic induction is followed by state-like changes in the organization of EEG functional connectivity in the theta and beta frequency bands in highhypnotically susceptible individuals. Front Hum Neurosci, 8. doi:ARTN 528 10.3389/fnhum.2014.00528

Jamieson, G. A., Dwivedi, P., \& Gruzelier, J. H. (2005). Changes in mismatch negativity across prehypnosis, hypnosis and post-hypnosis conditions distinguish high from low hypnotic susceptibility groups. Brain Res Bull, 67(4), 298-303. doi:10.1016/j.brainresbull.2005.06.033

Jensen, M. P., Adachi, T., \& Hakimian, S. (2015). Brain oscillations, hypnosis, and hypnotizability. American Journal of Clinical Hypnosis, 57(3), 230-253. doi:10.1080/00029157.2015.985573

Kallio, S. (1996). Harvardin hypnoosiherkkyysasteikko ryhmien arviointiin, Muoto A. [Finnish version of the Harvard Group Scale of Hypnotic Susceptibility, Form A, Shor and Orne, 1962]. The Society of Scientific Hypnosis in Finland.

Kallio, S., \& Ihamuotila, M. J. (1999). Finnish norms for the Harvard Group Scale of Hypnotic Susceptibility, Form A. International Journal of Clinical and Experimental Hypnosis, 47(3), 227-235.

Kallio, S., \& Koivisto, M. (2013). Posthypnotic suggestion alters conscious color perception in an automatic manner. International Journal of Clinical and Experimental Hypnosis, 61(4), 371387. doi:10.1080/00207144.2013.810446

Kallio, S., Koivisto, M., \& Kaakinen, J. K. (2017). Synaesthesia-type associations and perceptual changes induced by hypnotic suggestion. Scientific Reports, 7(1), 17310. doi:10.1038/s41598-017-16174-y

Kallio, S., Revonsuo, A., \& Lang, H. (2005). Can hypnosis and hypnotic hallucination change information processing in the brain - a case report. HYPNOS, XXXII(1), 25-35.

Kallio, S., Revonsuo, A., Lauerma, H., Hämäläinen, H., \& Lang, H. (1999). The MMN amplitude increases in hypnosis: a case study. Neuroreport, 10(17), 3579-3582.

Koivisto, M., Kirjanen, S., Revonsuo, A., \& Kallio, S. (2013). A preconscious neural mechanism of hypnotically altered colors: a double case study. PLoS One, 8(8), e70900. doi:10.1371/journal.pone.0070900 
Kujala, T., Tervaniemi, M., \& Schroger, E. (2007). The mismatch negativity in cognitive and clinical neuroscience: theoretical and methodological considerations. Biological Psychology, 74(1), 1-19. doi:10.1016/j.biopsycho.2006.06.001

Landry, M., Lifshitz, M., \& Raz, A. (2017). Brain correlates of hypnosis: A systematic review and metaanalytic exploration. Neuroscience \& Biobehavioral Reviews, 81(Pt A), 75-98. doi:10.1016/j.neubiorev.2017.02.020

Lee, J. S., \& Koo, B. H. (2012). Fractal analysis of EEG upon auditory stimulation during waking and hypnosis in healthy volunteers. International Journal of Clinical and Experimental Hypnosis, 60(3), 266-285. doi:10.1080/00207144.2012.675294

McGeown, W. J., Mazzoni, G., Venneri, A., \& Kirsch, I. (2009). Hypnotic induction decreases anterior default mode activity. Conscious Cogn, 18(4), 848-855. doi:10.1016/j.concog.2009.09.001

Morlet, D., Demarquay, G., Brudon, F., Fischer, C., \& Caclin, A. (2014). Attention orienting dysfunction with preserved automatic auditory change detection in migraine. Clinical Neurophysiology, 125(3), 500-511. doi:10.1016/j.clinph.2013.05.032

Näätänen, R., Gaillard, A. W., \& Mäntysalo, S. (1978). Early selective-attention effect on evoked potential reinterpreted. Acta Psychologica, 42(4), 313-329.

Näätänen, R., Paavilainen, P., Rinne, T., \& Alho, K. (2007). The mismatch negativity (MMN) in basic research of central auditory processing: a review. Clinical Neurophysiology, 118(12), 25442590. doi:10.1016/j.clinph.2007.04.026

Näätänen, R., Pakarinen, S., Rinne, T., \& Takegata, R. (2004). The mismatch negativity (MMN): towards the optimal paradigm. Clinical Neurophysiology, 115(1), 140-144.

Oakley, D. A., \& Halligan, P. W. (2009). Hypnotic suggestion and cognitive neuroscience. Trends Cogn Sci, 13(6), 264-270. doi:10.1016/j.tics.2009.03.004

Paavilainen, P., Cammann, R., Alho, K., Reinikainen, K., Sams, M., \& Naatanen, R. (1987). Eventrelated potentials to pitch change in an auditory stimulus sequence during sleep. Electroencephalography and Clinical Neurophysiology, Supplement, 40, 246-255.

Piccione, C., Hilgard, E. R., \& Zimbardo, P. G. (1989). On the degree of stability of measured hypnotizability over a 25-year period. Journal of Personality and Social Psychology, 56(2), 289-295.

Radtke, H. L., \& Spanos, N. P. (1981). Was I hypnotized?: a social psychological analysis of hypnotic depth reports. Psychiatry, 44(4), 359-376.

Radtke, H. L., \& Spanos, N. P. (1982). The effect of rating scale descriptors on hypnotic depth reports. Journal of Psychology, 111(2d Half), 235-245. doi:10.1080/00223980.1982.9915363

Rainville, P., Hofbauer, R. K., Bushnell, M. C., Duncan, G. H., \& Price, D. D. (2002). Hypnosis modulates activity in brain structures involved in the regulation of consciousness. Journal of Cognitive Neuroscience, 14(6), 887-901.

Rinne, T., Alho, K., Ilmoniemi, R. J., Virtanen, J., \& Näätänen, R. (2000). Separate time behaviors of the temporal and frontal mismatch negativity sources. Neuroimage, 12(1), 14-19. doi:10.1006/nimg.2000.0591

Sallinen, M., \& Lyytinen, H. (1997). Mismatch negativity during objective and subjective sleepiness. Psychophysiology, 34(6), 694-702.

Sams, M., Paavilainen, P., Alho, K., \& Naatanen, R. (1985). Auditory frequency discrimination and event-related potentials. Electroencephalography in Clinical Neurophysiology, 62(6), 437448.

Sculthorpe, L. D., Ouellet, D. R., \& Campbell, K. B. (2009). MMN elicitation during natural sleep to violations of an auditory pattern. Brain Research, 1290, 52-62. doi:10.1016/j.brainres.2009.06.013

Shor, R. E., \& Orne, E. C. (1962). Harvard group scale of hypnotic susceptibility, form A. Palo Alto, CA: Consulting Psychologists Press. 
Sussman. (2007). A new view on the MMN and attention debate - The role of context in processing auditory events. Journal of Psychophysiology, 21(3-4), 164-175. doi:10.1027/02698803.21.34.164

Sussman, Winkler, I., Huotilainen, M., Ritter, W., \& Näätänen, R. (2002). Top-down effects can modify the initially stimulus-driven auditory organization. Cognitive Brain Research, 13(3), 393-405.

Sussman, E. S. (2013). Attention matters: pitch vs. pattern processing in adolescence. Front Psychol, 4, 333. doi:10.3389/fpsyg.2013.00333

Terhune, D. B., Cleeremans, A., Raz, A., \& Lynn, S. J. (2017). Hypnosis and top-down regulation of consciousness. Neuroscience \& Biobehavioral Reviews, 81(Pt A), 59-74. doi:10.1016/j.neubiorev.2017.02.002

Tiainen, M., Tiippana, K., Paavilainen, P., Vainio, M., \& Vainio, L. (2017). Mismatch negativity (MMN) to speech sounds is modulated systematically by manual grip execution. Neuroscience Letters, 651, 237-241. doi:10.1016/j.neulet.2017.05.024

Vanhaudenhuyse, A., Laureys, S., \& Faymonville, M. E. (2014). Neurophysiology of hypnosis. Clinical Neurophysiology, 44(4), 343-353. doi:10.1016/j.neucli.2013.09.006

Williams, J. D., \& Gruzelier, J. H. (2001). Differentiation of hypnosis and relaxation by analysis of narrow band theta and alpha frequencies. International Journal of Clinical and Experimental Hypnosis, 49(3), 185-206. doi:10.1080/00207140108410070

Woldorff, M. G., Hillyard, S. A., Gallen, C. C., Hampson, S. R., \& Bloom, F. E. (1998). Magnetoencephalographic recordings demonstrate attentional modulation of mismatchrelated neural activity in human auditory cortex. Psychophysiology, 35(3), 283-292.

Yang, B., Xiao, W., Liu, X., Wu, S., \& Miao, D. (2013). Mental fatigue impairs pre-attentive processing: a MMN study. Neuroscience Letters, 532, 12-16. doi:10.1016/j.neulet.2012.08.080 


\section{Figures and Table}
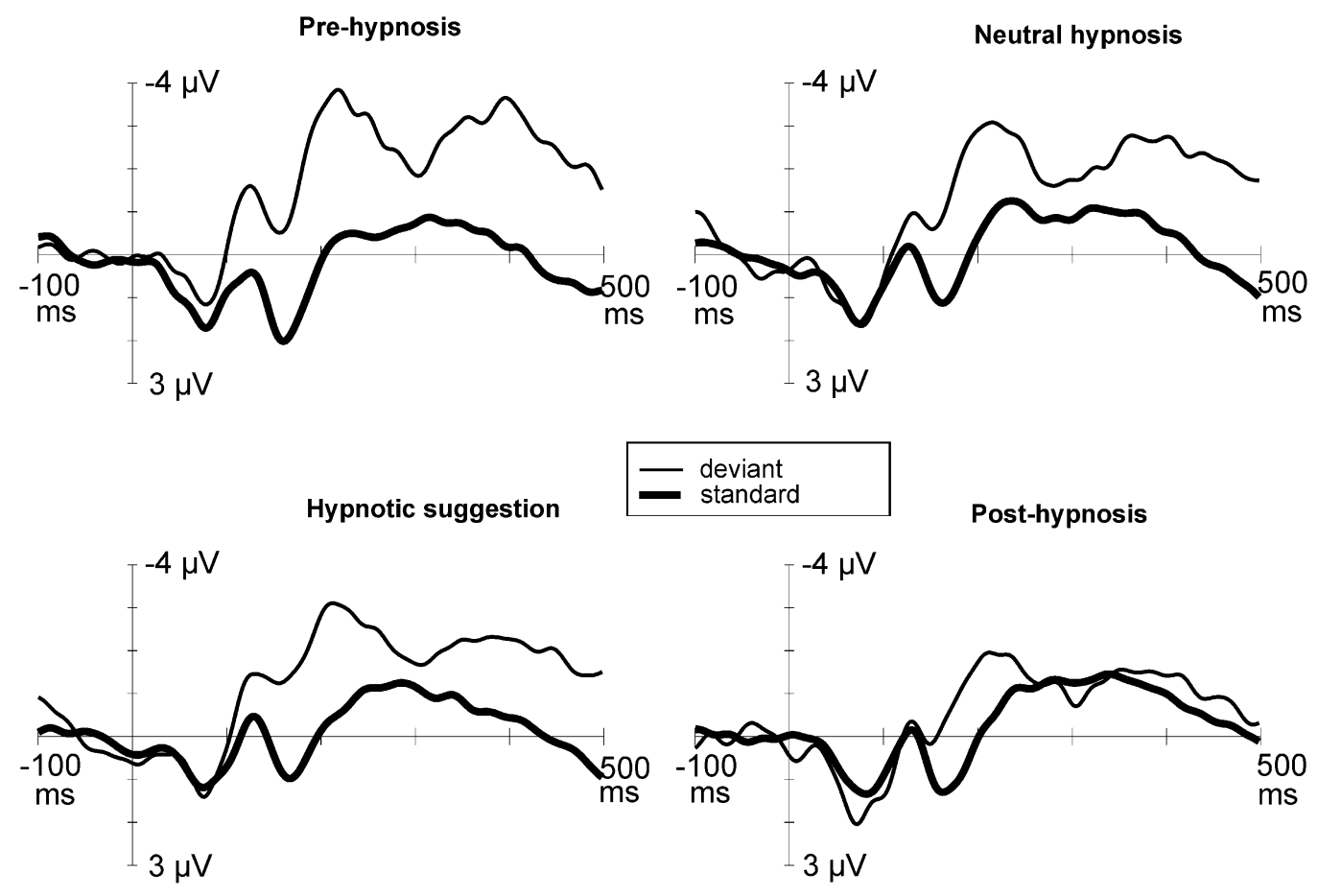

Figure 1. ERPs at Fz to deviant (thin line) and standard (thick line) stimuli in the four conditions $(\mathrm{N}=9)$. Negativity is plotted upwards. The window size of the interests is from $150 \mathrm{~ms}$ to $250 \mathrm{~ms}$.

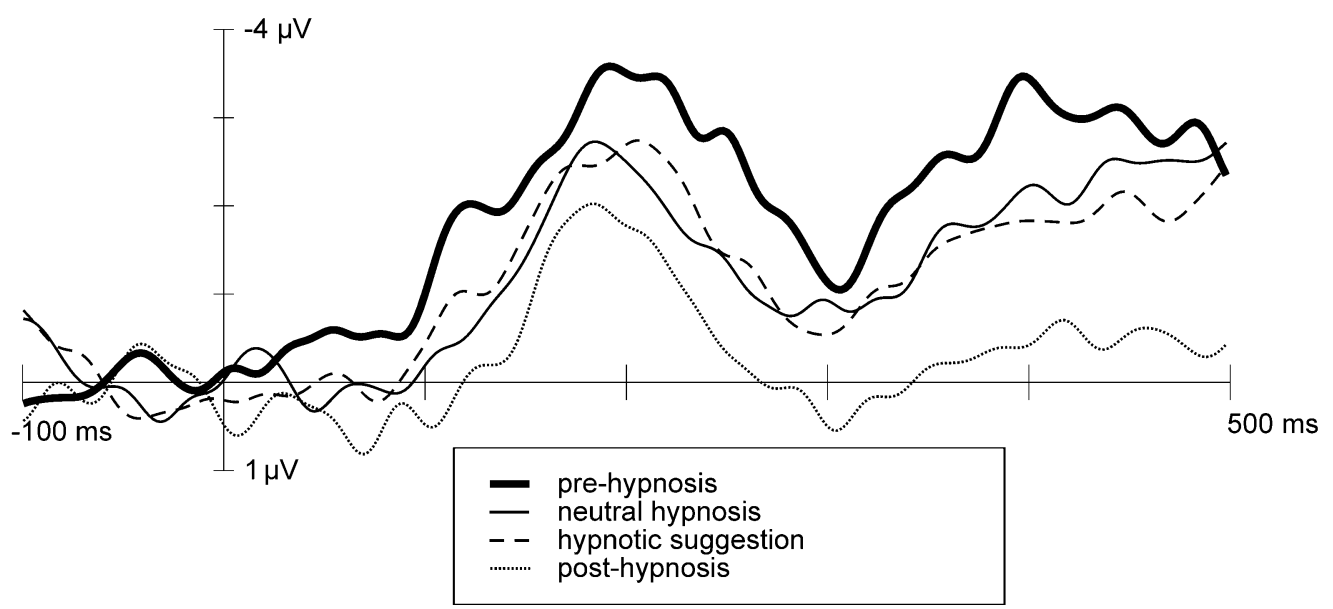

Figure 2. Deviant minus standard difference waves at $\mathrm{Fz}$ in the four conditions $(\mathrm{N}=9)$ 


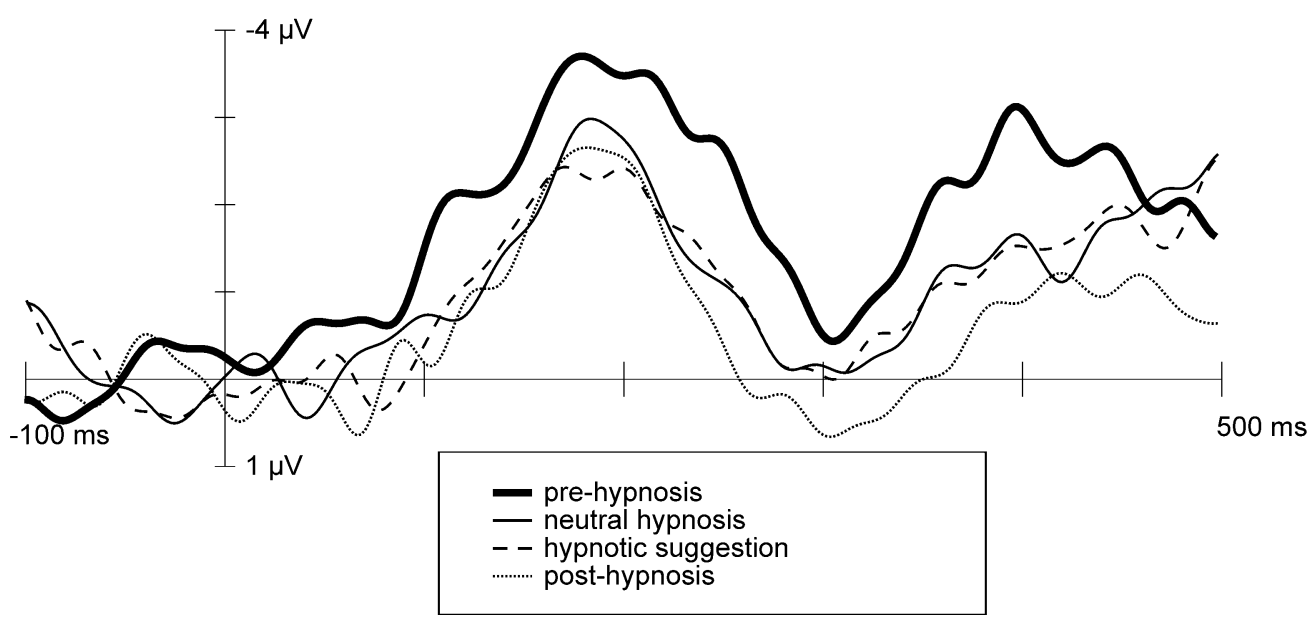

Figure 3. The deviant minus standard difference waves at $\mathrm{Fz}$ in the four conditions for those subjects $(\mathrm{N}=6)$ who had reported the fly suggestion as being real in the hypnotizability group measurement.

Table 1. Subjective hypnosis depth evaluations and their pairwise comparisons

\begin{tabular}{lllll}
\hline condition & mean depth (std) & condition 1 & condition 2 & p value \\
\hline PrH & $0.8(0.9)$ & PrH & HY & $<0.001$ \\
HY & $5.8(1.7)$ & & SU & 0.001 \\
SU & $5.7(2.7)$ & & PoH & ns \\
PoH & $0.9(1.3)$ & $\mathrm{HY}$ & $\mathrm{SU}$ & $\mathrm{ns}$ \\
& & & $\mathrm{PoH}$ & $<0.001$ \\
& & $\mathrm{SU}$ & $\mathrm{PoH}$ & $<0.001$ \\
\hline
\end{tabular}

$\operatorname{PrH}=$ Pre-hypnosis

$\mathrm{HY}=$ Neutral hypnosis

$\mathrm{SU}=$ Hypnotic suggestion

$\mathrm{PoH}=$ Post-hypnosis

ns $=$ not significant 